\title{
MAKING UP A SHORT GRB: THE BRIGHT FATE OF MERGERS OF COMPACT OBJECTS*
}

\author{
MIGUEL-ANGEL ALOY円 and P. MIMICA团 \\ Departamento de Astronomía y Astrofísica, Universidad de Valencia \\ 46100, Burjassot, Spain \\ Miguel.A.Aloy@uv.es
}

\begin{abstract}
We show some of the most important reasons why the likely fate of the merger of a neutron star with another compact object may be to yield a short gamma-ray burst (sGRB). Emphasis is made on some robust results that general relativistic (magneto)hydrodynamic simulations have established regarding the aforementioned subject.
\end{abstract}

\section{What do we know about the progenitor systems of sGRBs?}

Our knowledge about the progenitors of short gamma-ray bursts is relatively small. Any proposed progenitor system should be able of releasing $\sim 10^{49} \mathrm{erg}$ in the form of thermal energy or Poynting flux. Furthermore, the progenitor is requested to yield outflows collimated into cones of half-opening angle $\theta_{\mathrm{j}} \sim 4^{\circ}-25^{\circ}$, as inferred from the observed breaks in the light curves of some short GRBs, ${ }^{6}$ or the lower limits on $\theta_{\mathrm{j}}$ deduced from the absence of such breaks. ${ }^{7,10}$ Additionally, flow variability down to a few milliseconds over timescales $\sim 0.1-2 \mathrm{~s}$ has to be produced. Also, any viable progenitor has to satisfy the fact that it can be generated in any of the typical hosts observed for a handful of burst, ${ }^{16}$ as well as to occur at rates equal or larger than the rate at which sGRBs are produced.

\section{Why are mergers of compact objects good candidates to be progenitors of sGRBs?}

A family of systems that has been proposed as likely progenitors of sGRBs is the remnant left by the merger of a binary system of compact objects formed by either two neutron stars (NSs) or a NS and a BH. ${ }^{15}$ Such systems consist of a BH girded by a thick gas torus from which it swallows matter at a hypercritical rate. In this situation the cooling is dominated by the emission of neutrinos. These neutrinos might either be the primary energy source blowing a fireball of $\mathrm{e}^{+} \mathrm{e}^{-}$pairs and photons or to load with pairs a Poynting dominated outflow. ${ }^{13}$ The duration of the produced outflows is, in part, limited by the time needed by the $\mathrm{BH}$ to engulf most of the matter of the accretion disk, namely, a few $100 \mathrm{~ms}$ (but see below). Thereby, a merger of compact objects (MCO) may release energy during a sufficiently long time to fuel a short duration sGRB event. This limit on the time scale, set by the ON time of the source, holds for both $\nu$-powered jets and for MHD-generated outflows. ${ }^{4}$

\footnotetext{
*This research has been partially supported by the Spanish Ministerio de Ciencia y Tecnología grant AYA2001-3490-C02-C01 and through the EU grant MEIF-CT-2005-009395.

${ }^{\dagger}$ Ramón y Cajal Fellow of the Spanish Ministry of Education and Science

${ }^{\ddagger}$ Marie Curie Fellow
} 
According to the state-of-the-art numerical simulations of MCOs including realistic microphysics ${ }^{12}$ it is possible to release a few $10^{49} \mathrm{erg}$ above the poles of a stellar mass $\mathrm{BH}$ in a region of nearly vacuum as a result of the process of $\nu \bar{\nu}$-annihilation in such region. Detailled simulations of such process suggest that even more energy could be deposited in the system, ${ }^{5}$ although time-dependent numerical models including energy transport are needed to give more reliable numbers. Therefore, MCOs provide an energy budged which may satisfy the energy requirements to produce sGRBs. Numerical simulations have also shown the ability of the system to tap the energy from the $\mathrm{BH}$ and potentially fuel a GRB. ${ }^{14}$

In the standard merger scenario, initially the spiral-in process may take $\sim 1 \mathrm{~Gy}$. Since it is likely that the newly born binary system may receive a natal kick (of a few $\sim 100 \mathrm{~km} \mathrm{~s}^{-1}$ ), it is expected that, when the two compact objects merge, they have travelled to the outer skirts of the galaxy or, even, to the intergalactic medium. This evolutionary path is compatible with observing sGRBs outside of their putative host galaxies (e.g., GRB 050813). ${ }^{16}$ Alternatively, the evolutionary tracks of MCOs might be much shorter $(\sim 1 \mathrm{My}),{ }^{18}$ which has the implication that many mergers could happen inside of their hosts galaxies and, hence, this would accommodate the small offsets with respect to the galactic center observed for a few sGRBs (e.g., GRB 050724). ${ }^{16}$

The number of well identified galaxies hosting sGRBs is still small to have a good statistical sample. Nevertheless, it seems that sGRBs are associated with both (young) start-forming and (old) elliptical galaxies. ${ }^{8,16}$ It is very appealing (but still quite speculative) to establish an association between sGRBs detected in old galaxies with mergers happening after long evolutionary paths and, on the other side, between sGRBs found in young galaxies with faster evolving mergers. However, taking together the information provided by the typical galactic offsets and the typical host galaxies of sGRBs one may infer only circumstantial evidences about the nature of the progenitors of sGRBs.

Self-consistent (magneto)hydrodynamic modeling is needed to address issues like:

Collimation. The generated outflows are either collimated by the accretion disk $^{3}$ or self-collimated by the magnetic field. ${ }^{14}$ The typical outflow half-opening angles are $\sim 3^{\circ}-25^{\circ}$ (i.e., compatible with observations; see $\S 1$ ). The baryon-poor outflows develop a transverse structure. Particularly, the transverse profile of the Lorentz factor could be roughly fit by a Gaussian function, but more complicated functions are required to provide accurate fits. ${ }^{3}$

Variability. Even injecting energy at constant rates, the produced outflows are highly variable. The interaction of the newborn fireball with the accretion torus yields the growth of Kelvin-Helmholtz ${ }^{3}$ instabilities. In case of MHD jets the variability is imprinted by pinch instabilities. ${ }^{14}$ All computed axisymmetric models seem to be either stable or marginally stable. It is not yet numerically verified whether $3 \mathrm{D}$ jets emerging from hyperaccreting BHs are stable.

Influence of the environment. Mergers in low density environments may fuel 
ultrarelativistic outflows with the potential to produce normal sGRBs, while in case that the merger occurs in high density media, the observational signature is not a sGRB. ${ }^{3}$ The fact that depending on the environmental density an sGRB can be produced or not has direct implications for the estimates ${ }^{9}$ of the true rates of sGRBs and compared with the rates of NS+NS mergers.

Asymptotic Lorentz factor. Saturation values of the bulk Lorentz factor, $\Gamma_{\infty} \gtrsim 500-1000$ are obtained for both thermally ${ }^{3}$ and magnetically ${ }^{14}$ generated outflows. For thermally produced outflows, there is a trend to produce much higher values of $\Gamma_{\infty}$ for sGRBs $\left(\Gamma_{\infty} \gtrsim 500-1000\right)$ than for lGRBs $\left(\Gamma_{\infty} \sim 100\right)$. This difference in Lorentz factor might be the reason for the paucity of soft sGRBs. ${ }^{11}$

Duration of the events. In addition to the ON time of the source, the other factor that sets the duration of a GRB event is the radial stretching of the fireball which results from the differential acceleration of the forward and rear edges of the fireball. ${ }^{3,11}$ A prolonged activity could also be produced by the fall back onto the $\mathrm{BH}$ of a fraction of the matter ejected during the early stages of the merger. ${ }^{17}$

\section{Acknowledgments}

It is a pleasure to thank H.-Th Janka and E. Müller for encouragement and support. The author thanks Max-Planck-Institut für Astrophysik for hospitality. This work has been partially supported by SFB 375 "Astroparticle Physics" and SFB-TR 7 "Gravitational Wave Astronomy" of the Deutsche Forschungsgemeinschaft.

\section{References}

1. M. A. Alloy, et. al., ApJL 531, L119 (2000).

2. M. A. Aloy, J. M. Ibáñez, J.-A. Miralles and V. Urpin, A $\& A$ 396, 693 (2002).

3. M. A. Aloy, H.-Th. Janka and E. Müller, ApJ 436, 273 (2005).

4. M. A. Aloy and M. Obergaulinger, astro-ph/0701187

5. R. Birkl, M. A. Aloy, H.-Th. Janka and E. Mueller, astro-ph/0608543

6. D. N. Burrows, et. al., ApJ, 653, 468 (2006)

7. De Ugarte Postigo, A., et al., ApJL, 648, 83 (2006)

8. D. B. Fox, et. al., Nat. 437, 845 (2005).

9. D. Guetta and T. Piran, ApJ 435, 421 (2005).

10. D. Grupe, et. al., ApJ, 653, 462 (2006)

11. H.-Th. Janka, M. A. Aloy, P. A. Mazzali and E. Pian, ApJ 645, 1305 (2006).

12. H.-Th. Janka et. al., ApJ 527, L39 (1999); M. Ruffert and H.-Th. Janka, ApJ 344, 573 (1999); S. Rosswog and E. Ramirez-Ruiz, MNRAS 336, L7 (2002); S. Rosswog, E. Ramirez-Ruiz and M. B. Davies, MNRAS 345, 1077 (2003); R. Oechslin and H.-Th. Janka, MNRAS 368, 1489 (2006).

13. A. Levinson and D. Eichler, ApJ 418, 386 (1993).

14. J. C. McKinney, MNRAS 368, 1561 (2006)

15. B. Paczynski, ApJL 308, L43 (1986); J. Goodman, ApJL 308, L47 (1986); D. Eichler, et. al., Nat. 340, 126 (1989); R. Mochkovitch, et. al., Nat. 361, 236 (1993).

16. J. X. Prochaska, et. al., ApJ, 642, 989 (2006)

17. S. Rosswog, astro-ph/0611440

18. A. V. Tutukov and L. R. Yungelson, MNRAS 260, 675 (1993); K. Belczynski, V. Kalogera and T. Bulik, ApJ bf 572, 407 (2002). 